\title{
Boosting Terahertz Generation in Laser-Field Ionized Gases Using a Sawtooth Wave Shape
}

\author{
P. González de Alaiza Martínez, ${ }^{1, *}$ I. Babushkin, ${ }^{2}$ L. Bergé, ${ }^{1}$ S. Skupin, ${ }^{3}$ E. Cabrera-Granado, ${ }^{4}$ C. Köhler, ${ }^{5}$ \\ U. Morgner, ${ }^{2}$ A. Husakou, ${ }^{6}$ and J. Herrmann ${ }^{6}$ \\ ${ }^{1}$ CEA-DAM, DIF, F-91297 Arpajon, France \\ ${ }^{2}$ Institute of Quantum Optics, Leibniz University Hannover, Welfengarten 1 30167, Hannover, Germany \\ ${ }^{3}$ Univ. Bordeaux - CNRS - CEA, Centre Lasers Intenses et Applications, UMR 5107, 33405 Talence, France \\ ${ }^{4}$ Facultad de Óptica y Optometría, Universidad Complutense de Madrid, 28037 Madrid, Spain \\ ${ }^{5}$ Institute for Scientific Computing, TU Dresden, 01062 Dresden, Germany \\ ${ }^{6}$ Max-Born-Institut für Nichtlineare Optik und Kurzzeitspektroskopie, 12489 Berlin, Germany
}

(Received 7 December 2014; published 6 May 2015)

\begin{abstract}
Broadband ultrashort terahertz $(\mathrm{THz})$ pulses can be produced using plasma generation in a noble gas ionized by femtosecond two-color pulses. Here we demonstrate that, by using multiple-frequency laser pulses, one can obtain a waveform which optimizes the free electron trajectories in such a way that they acquire the largest drift velocity. This allows us to increase the $\mathrm{THz}$ conversion efficiency to $2 \%$, an unprecedented performance for $\mathrm{THz}$ generation in gases. In addition to the analytical study of $\mathrm{THz}$ generation using a local current model, we perform comprehensive 3D simulations accounting for propagation effects which confirm this prediction. Our results show that $\mathrm{THz}$ conversion via tunnel ionization can be greatly improved with well-designed multicolor pulses.
\end{abstract}

DOI: 10.1103/PhysRevLett.114.183901

PACS numbers: 42.65.Re, 32.80.Fb, 52.50.Jm

Ultrashort pulses in the terahertz $(\mathrm{THz})$ range (from $\sim 0.1$ to $\sim 30 \mathrm{THz}$ ) are extremely important for various timeresolved studies in molecular physics, chemistry, material sciences, and security applications [1-8]. One of the major challenges in this field is the development of $\mathrm{THz}$ emitters producing high peak intensities. So far, besides conventional devices such as antennas, photoconductive switches, etc., two main techniques have been explored for producing subps THz pulses with energies in the microjoule range. The first method is based on optical rectification in second-order nonlinear crystals [9]. Pumped by multi-mJ single color pulses, this technique requiring phase matching can generate $\mathrm{THz}$ pulses with $10 \mu \mathrm{J}$ energy, but the bandwidth is limited to a few THz. Recently, large-sized organic crystals were used to deliver $\mathrm{THz}$ pulses with $\mathrm{GV} / \mathrm{m}$ electric field strength, and a conversion efficiency of about $1 \%$ was demonstrated [10]. The second method is based on focusing a femtosecond pulse together with its second harmonic into a gas cell and create a plasma [11-24]. In such two-color pump setup, free electrons produced by tunnel ionization acquire a nonzero drift velocity and generate a quasi-dc current which is responsible for $\mathrm{THz}$ emission [19]. The mechanism underlying $\mathrm{THz}$ generation in gases is intrinsically related to the optically induced stepwise increase of the free electron density near the extrema of the ionizing optical field $[15,16,22]$. An easy explanation is that the Fourier transform of a steplike function in time has its maximum near the zero frequency. Besides, critical for the generated $\mathrm{THz}$ energy is a pronounced asymmetry in time of the pump wave shape with respect to the field extrema, which dictates the electron drift velocity. This gas-based scheme for $\mathrm{THz}$ generation provides higher breakdown threshold and broader spectral ranges than the method involving crystals [11-13,17-20]. THz pulses in gases with high field strength $>1 \mathrm{GV} / \mathrm{m}$ were simulated in [16]; however, the highest reported $\mathrm{THz}$ energies $(5 \mu \mathrm{J})$ correspond to conversion efficiencies of about $10^{-4}$ only.

In this Letter, we exploit degrees of freedom given by appropriately chosen pump waveforms beyond a simple two-color field to increase the $\mathrm{THz}$ conversion efficiency. We show that fields with a sawtoothlike temporal shape do promote the highest $\mathrm{THz}$ signals triggered by tunnelinduced photocurrents. We predict, by means of a local theory, that such waveforms can, in principle, increase the $\mathrm{THz}$ efficiency by up to 2 orders of magnitude, compared to a standard two-color pulse. Qualitatively, for a sawtoothlike optical waveform, the asymmetry at the field extrema is maximized, leading to a dramatically enhanced conversion efficiency. Boosting the $\mathrm{THz}$ yield is confirmed through comprehensive $3 \mathrm{D}$ computations that take all propagation effects into account. Selecting the first four Fourier harmonics of the sawtooth waveform already guarantees an impressive increase of the THz energy up to $5 \mu \mathrm{J}$ from a $300 \mu \mathrm{J}$ pump pulse.

We start with the local current (LC) approximation [15], which neglects propagation effects. The free electron density $\rho(t)$ and current $J(t)$ are governed by

$$
\frac{\partial \rho(t)}{\partial t}=W[E(t)]\left[\rho_{0}-\rho(t)\right]
$$



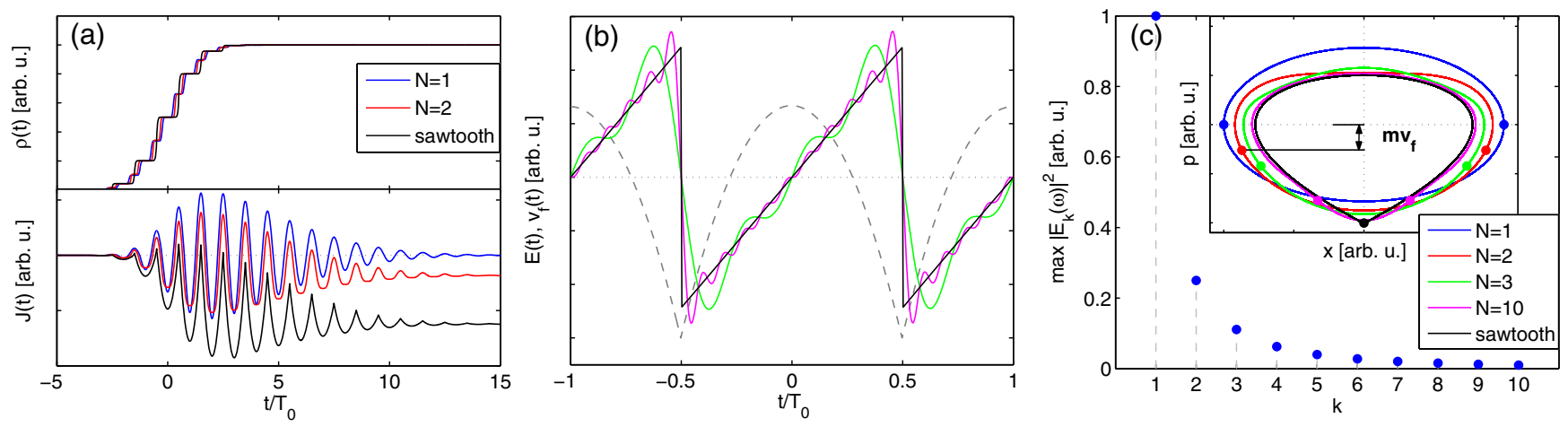

FIG. 1 (color online). (a) (top) Stepwise increase of the electron density $\rho(t)$ and (bottom) current density $J(t)$ for a one-color, twocolor, and a sawtooth pulse with identical ionization yield in argon. The sawtooth pulse develops a larger low-frequency component in $J$. (b) Sawtooth waveform $E(t)$ (black solid line) having the maxima of $\left|v_{f}(t)\right|$ (gray dashed line) at the same instants as the extrema of $E(t)$. Colored solid curves show the three-color (green curve) and ten-color (magenta curve) approximations to the ideal sawtooth shape. (c) Spectrum of the sawtooth waveform containing all harmonics of $\omega_{0}$ with intensities decreasing as $1 / k^{2}$. Inset shows the trajectories of free electrons in the phase space $(p, x)$ for an increasing number of colors. Dots locate the maxima of $|E(t)|$; for the two-color case, the value of $m v_{f}$ at those maxima is exemplified.

$$
\frac{\partial J(t)}{\partial t}=\frac{q^{2}}{m} \rho(t) E(t)-\frac{J(t)}{\tau_{c}},
$$

where $E(t)$ is the pump field;

$$
W[E(t)]=\frac{\alpha}{|E|} \exp \left[-\frac{\beta}{|E|}\right]
$$

is the instantaneous tunnel ionization rate [13], $\rho_{0}$ is the density of neutral atoms, $q$ and $m$ are the charge and mass of the electron, and $\tau_{c}$ is the current decay time due to collisions. In Eq. (3), $\alpha=4 \omega_{a}\left(r_{H}\right)^{5 / 2} E_{a}$ and $\beta=$ $2\left(r_{H}\right)^{3 / 2} E_{a} / 3$ depend on the ratio of the ionization potential $U_{i}$ of the considered gas over the hydrogen ionization potential, $r_{H}=U_{i} / U_{H}$, while $E_{a}=m^{2} q^{5} /\left(4 \pi \varepsilon_{0}\right)^{3} \hbar^{4}$ and $\omega_{a}=m q^{4} /\left(4 \pi \varepsilon_{0}\right)^{2} \hbar^{3}$.

The THz field $E_{\mathrm{THz}}(t)$ is generated by the free electrons created by tunnel ionization and then accelerated in the pump field. For pump fields with a temporal asymmetry around the ionization instants, the current density $J(t)$ develops a low-frequency component following the stepwise increase of the electron density [see Fig. 1(a)]. Assuming a small size of the plasma spot, $E_{\mathrm{THz}}(t)$ is evaluated by the low-frequency filtering of $g \partial_{t} J(t)$, where $g$ is a geometrical factor [15]. Ionization mostly happens near the extrema of $E(t)$. In the following, we number their corresponding instants consecutively as $t_{1}, t_{2}, t_{3}, \ldots, t_{n}$. Thus, the electron density and current [Eqs. (1) and (2)] can be approximated as follows (see [15] for details):

$$
\begin{gathered}
\rho(t) \simeq \sum_{n} \delta \rho_{n} H_{n}\left(t-t_{n}\right), \\
J(t) \simeq J_{A}(t)+J_{B}(t), \\
J_{A}(t)=\sum_{n} q \delta \rho_{n} v_{f}(t) H_{n}\left(t-t_{n}\right), \\
J_{B}(t)=-\sum_{n} q \delta \rho_{n} e^{-\left[\left(t-t_{n}\right) / \tau_{c}\right]} v_{f}\left(t_{n}\right) H_{n}\left(t-t_{n}\right),
\end{gathered}
$$

where $v_{f}(t)$ is the "free electron velocity" [25] given by

$$
v_{f}(t)=\frac{q}{m} e^{-\left(t / \tau_{c}\right)} \int_{-\infty}^{t} E\left(t^{\prime}\right) e^{\left(t^{\prime} / \tau_{c}\right)} d t^{\prime}
$$

This quantity determines the net nonzero drift velocity which ionized electrons, born with zero velocity at $t=t_{n}$, retain after the laser pulse has passed. The smoothed step function used in Eqs. (4)-(7) is $H_{n}(t)=\frac{1}{2}\left[1+\operatorname{erf}\left(t / \tau_{n}\right)\right]$, where $\tau_{n}$ is the width of the $n$th ionization event and the density jump at $t=t_{n}, \delta \rho_{n}$, is expressed as (see Supplemental Material [26])

$$
\delta \rho_{n} \simeq \rho_{0} \epsilon_{n}\left(1-e^{-\sqrt{\pi} W\left[E\left(t_{n}\right)\right] \tau_{n}}\right),
$$

with $\epsilon_{1}=1$ and $\epsilon_{n}=e^{-\sqrt{\pi} \sum_{j=1}^{n-1} W\left[E\left(t_{j}\right)\right] \tau_{j}}$ for $n>1$. In Eq. (7), $v_{f}\left(t_{n}\right)$ contains the information on both the asymmetry and shape of the optical pulse.

Decomposing the current $J$ into $J_{A}$ and $J_{B}$ allows us to separate the two different frequency scales: the current $J_{A}$ contains mainly the high frequency pump spectrum, whereas the current $J_{B}$ contains the $\mathrm{THz}$ spectrum related to the ionization events at times $t=t_{n}$. It is possible to estimate analytically the spectrum of the $\mathrm{THz}$ radiation generated by the current components $J_{A}$ and $J_{B}$. Using Fourier transforms, we obtain in the low-frequency domain (see Supplemental Material [26] for a detailed derivation)

$$
\begin{aligned}
\mathcal{F}\left[\partial_{t} J_{B}\right](\omega) & \approx \frac{-q}{\sqrt{2 \pi}} \sum_{n} \delta \rho_{n} v_{f}\left(t_{n}\right) e^{i t_{n} \omega} \frac{\omega}{i / \tau_{c}+\omega}, \\
\mathcal{F}\left[\partial_{t} J_{A}\right](\omega) & \approx \frac{-i q^{2}}{\sqrt{2 \pi} m} \sum_{n} \sum_{k=1}^{N} \delta \rho_{n} E_{k}\left(t_{n}\right) \frac{\omega}{k^{2} \omega_{0}^{2}} .
\end{aligned}
$$

Here, to evaluate $J_{A}(t)$, we considered multicolor pulses of the form 


$$
E(t)=\sum_{k=1}^{N} E_{k}(t)=\sum_{k=1}^{N} \mathcal{E}_{k}(t) a_{k} \cos \left(k \omega_{0} t+\phi_{k}\right),
$$

where $\mathcal{E}_{k}(t), a_{k}$, and $\phi_{k}$ are the envelope with duration $\tau_{k}$, relative amplitude and phase of the $k$ th harmonic, respectively; $\omega_{0}$ is the fundamental frequency and we assumed long pulses $\omega_{0} \tau_{k} \gg 1$. For a near-infrared pump, the contribution $J_{B}$ dominates over $J_{A}[15,21]$ in the $\mathrm{THz}$ spectral range, because the $E_{k}\left(t_{n}\right)$ are not sign definite and the summands in Eq. (10b) mostly cancel each other, which we verified for our sawtooth shapes. We therefore neglect $J_{A}$ in the coming analysis and henceforth assume Gaussian envelopes $\mathcal{E}_{k}(t)=E_{0} e^{-t^{2} / \tau^{2}}$ with amplitude $E_{0}$ and duration $\tau$ being identical for all colors.

The energy in the $\mathrm{THz}$ part of the spectrum below a certain cutoff frequency $\omega_{\text {co }} \gg 1 / \tau_{c}$ can be thus estimated by the local $\mathrm{THz}$ yield as $U_{\mathrm{THz}} \propto \int_{0}^{\omega_{\mathrm{co}}}\left|\omega \hat{J}_{B}(\omega)\right|^{2} d \omega$ $\propto\left[\sum_{n} \delta \rho_{n} v_{f}\left(t_{n}\right)\right]^{2}$. Obviously, to maximize $U_{\mathrm{THz}}$ one may try to increase not only the ionization yield $\delta \rho_{n}$, but also the free electron velocity $v_{f}\left(t_{n}\right)$. Thus, maximizing the $\mathrm{THz}$ energy requires that $v_{f}(t)$ reaches an extremum at every discrete instant $t_{n}$, and that all $v_{f}\left(t_{n}\right)$ have the same sign.

According to Eq. (8), $v_{f}(t)$ attains local extrema when $E(t)$ changes its sign. So the only way to achieve maxima of both $|E(t)|$ and $\left|v_{f}(t)\right|$ at the same instants is to have a discontinuous electric field. This condition is fulfilled by the sawtooth waveform, which can be obtained by setting $a_{k}=1 / k$ and $\phi_{k}=(-1)^{k} \pi / 2$ in Eq. (11). The sawtooth waveform and $v_{f}(t)$ are illustrated by Fig. 1(b) in the limit of negligible free electron collisions, $\tau_{c} \rightarrow+\infty$. Figure 1(b) also shows that the velocities $v_{f}\left(t_{n}\right)$ are sign definite.

Practically, as an infinite number of harmonics in the pump is not achievable, we should employ a finite number $N$ of colors. As shown in Fig. 1(b), the sawtooth shape is fairly well reproduced for $N \geq 3$, which is confirmed by the sawtooth spectrum of Fig. 1(c). The inset of this figure details the free electron phase space $p(t) \propto \int_{-\infty}^{t} E\left(t^{\prime}\right) d t^{\prime} \sim$ $m v_{f}(t)$ versus $x(t)$, as well as the maximum values of $|E(t)|$ (see dots). One can see that the sign-definite value of $p(t)$ at the field extrema indeed increases with the number of colors, thus increasing $U_{\mathrm{THz}}$.

In the following, we fix $\omega_{0}$ corresponding to the wavelength $\lambda_{0}=1600 \mathrm{~nm}$, a choice clearly advantageous over the usual one $\lambda_{0}=800 \mathrm{~nm}$, because more harmonics are accessible in practice. In particular, the first four harmonics are $\lambda_{0} / 2=800 \mathrm{~nm}, \lambda_{0} / 3=533 \mathrm{~nm}$, and $\lambda_{0} / 4=400 \mathrm{~nm}$. All these wavelengths can be produced from an $800 \mathrm{~nm}$ femtosecond laser source using, for instance, optical parametric amplification to obtain $\lambda_{0}$ and $\lambda_{0} / 3$ and frequency doubling to obtain $\lambda_{0} / 4$. In contrast, for $\lambda_{0}=800 \mathrm{~nm}$ the fourth harmonic at $200 \mathrm{~nm}$ lies already in the UV and is not so easy to produce. Throughout the Letter, we consider argon at 1 atm pressure and assume a Gaussian pulse envelope with $40 \mathrm{fs}$ FWHM duration $(\tau=34 \mathrm{fs})$.
Let us first check our analytical predictions by computing numerically the local $\mathrm{THz}$ yield $U_{\mathrm{THz}}$ in the frequency range $\nu \equiv \omega / 2 \pi<\nu_{\mathrm{co}} \equiv \omega_{\mathrm{co}} / 2 \pi=100 \mathrm{THz}$ from the LC model. For a given multicolor pulse [Eq. (11)] we compute the current $J(t)$ according to Eqs. (1)-(3) and evaluate the local THz yield in Fourier space $U_{\mathrm{THz}} \propto \int_{0}^{\nu_{\mathrm{co}}}|\nu \hat{J}(\nu)|^{2} d \nu$ [27]. From our coming 3D simulations accounting for propagation effects we know that for given pulse energy and focusing conditions the ionization yield for different multicolor configurations is almost the same [see Fig. 3(d)]. This is understandable, because free electrons have a strong defocusing effect and balance the intensity growth in the focal region, similar to the well-known intensity clamping in femtosecond filaments [28]. A reasonable strategy is thus to compare the local $\mathrm{THz}$ yield from pulses producing a constant ionization level controlled by $\rho(t \rightarrow+\infty)=\rho^{\max }$ in Eq. (1)

Figure 2 summarizes results from the LC model Eqs. (1)-(3) for Gaussian multicolor pulses with ionization yield $\rho^{\max }$ fixed to $10 \%$ of $\rho_{0}$. For given $a_{k}$ and $\phi_{k}$ the field amplitude $E_{0}$ is chosen such that $\rho^{\max }=2.7 \times 10^{18} \mathrm{~cm}^{-3}$. First, we fix relative amplitude and phase for the fundamental frequency to $a_{1}=1, \phi_{1}=-\pi / 2$. This choice is arbitrary, because for multicycle pulses $(\tau=34 \mathrm{fs})$
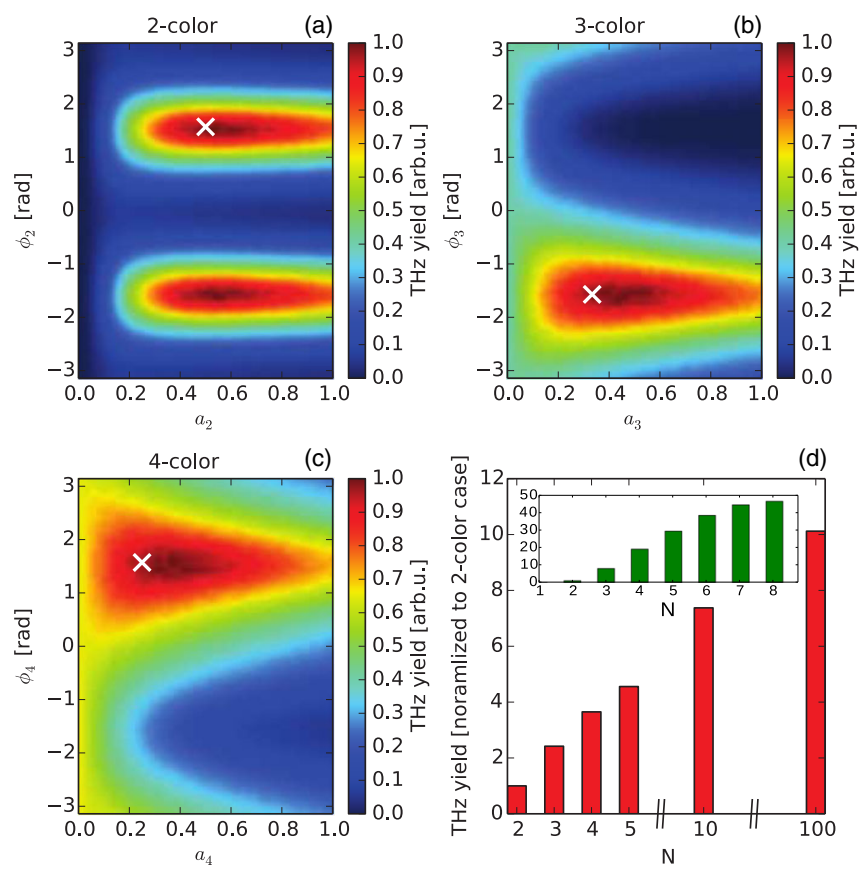

FIG. 2 (color online). (a) Dependency of local THz yield on $a_{2}, \phi_{2}$ for a $N=2$ color field in Eq. (11). (b) Same for $a_{3}, \phi_{3}$ and $N=3$. (c) Same for $a_{4}, \phi_{4}$ and $N=4$. Relative amplitude and phases of the lower harmonics in (a)-(c) are fixed according to the (optimum) sawtooth shape, i.e., $a_{k}=1 / k, \phi_{k}=(-1)^{k} \pi / 2$. The total electric field amplitude $E_{0}$ is determined by the fixed ionization yield $\rho^{\max }=2.7 \times 10^{18} \mathrm{~cm}^{-3}$. White crosses in (a)-(c) indicate the values of the sawtooth waveform. (d) $N$-color sawtooth THz yield normalized to the two-color pulse one when $\rho^{\max }$ is fixed. For comparison, the inset shows the THz yield for fixed pump energy flux $E_{0}^{2} \sum_{k} a_{k}^{2}$. 
carrier-envelope phase effects are negligible. For $N=4$ colors, we are then left with six free parameters, $a_{2}, a_{3}, a_{4}$ and $\phi_{2}, \phi_{3}, \phi_{4}$. Because we cannot visualize the dependency of the $\mathrm{THz}$ yield on all six parameters in the same figure, we treat two-, three-, and four-color cases separately and vary the relative amplitude and phase of the highest harmonic only [see Figs. 2(a)-2(c)]. Clearly, we observe maximum $\mathrm{THz}$ yield very close to the $N$ th-order truncation of the ideal sawtooth waveform in all cases. We checked that this behavior does not change when one selects the ionization level to $5 \%$ or $50 \%$ of $\rho_{0}$. An important point is also the stability of the resulting yield against small variations in the laser parameters, which are expected in a realistic setup. From Figs. 2(a)-2(c) it is clear that fluctuations in phases or amplitudes up to $\sim 10 \%$ do not change the $\mathrm{THz}$ yield significantly.

An interesting issue is how the overall $\mathrm{THz}$ signal depends on the number of harmonics that approximates the sawtooth shape. We clarify this question in Fig. 2(d). One can see that the $\mathrm{THz}$ yield significantly increases until $N \sim 5$, while its quasilinear growth saturates for larger $N$. This behavior is also supported analytically in the Supplemental Material [26]. There, we also justify that the pump wave shape that optimizes the $\mathrm{THz}$ yield approaches the sawtooth one at large $N$.

In the inset of Fig. 2(d) we find it instructive to present the efficiency of the $N$-color sawtooth approximation in the LC limit when the pump energy flux $E_{0}^{2} \sum_{k} a_{k}^{2}$ is fixed, instead of preserving the same ionization level. In this case, the sawtooth shape allows us to achieve a more impressive boost up to a factor of 50 , because not only $v_{f}\left(t_{n}\right)$ but also $\rho_{n}$ grow considerably. Remarkably, somewhat similar wave shapes were found to increase the yield and electron recollision energy in the high-order harmonic generation process by up to 2 orders of magnitude $[29,30]$.

The advantage of the four-color approximation of a sawtooth field is now studied using the unidirectional pulse propagation equation that takes into account propagation effects in full space and time resolved geometry. This 3D model was successfully tested against experimental data for $\mathrm{THz}$ generation from two-color pulses [22]. We use an adapted version of the unidirectional pulse propagation equation [31] for linearly polarized pulses

$$
\partial_{z} \hat{E}=i \sqrt{k^{2}(\omega)-k_{x}^{2}-k_{y}^{2}} \hat{E}+i \frac{\mu_{0} \omega^{2}}{2 k(\omega)} \hat{P}_{\mathrm{NL}} .
$$

Here, $\hat{E}\left(k_{x}, k_{y}, z, \omega\right)$ is the pulse electric field expressed in the Fourier domain with respect to transverse coordinates and time, $k=\omega n(\omega) / c$ is the wave number, $c$ is the speed of light, and $n(\omega)$ is the linear refractive index of argon [32]. The nonlinear polarization $\hat{P}_{\mathrm{NL}}(\omega)=\hat{P}_{\mathrm{Kerr}}(\omega)+$ $i \hat{J}(\omega) / \omega+i \hat{J}_{\text {loss }}(\omega) / \omega$ accounts for third-order nonlinear polarization $P_{\text {Kerr }}(t)$, electron current $J(t)$, and a loss term $J_{\text {loss }}(t)$ due to photon absorption during ionization. The plasma current $J(t)$ is described by Eqs. (1)-(3). Since 3D propagation affects relative phases, local intensities, and pulse durations, we can anticipate a reduced $\mathrm{THz}$ conversion efficiency compared with the prediction of Fig. 2(d).

Figure 3(a) displays the pulse spectrum (left axis and colorbar) and the THz yield below $100 \mathrm{THz}$ (right axis) of a two-color 40-fs pulse with overall energy of $300 \mu \mathrm{J}$. The fundamental wavelength is $800 \mathrm{~nm}$ and $6 \%$ of the pulse energy is in the second harmonic ( $\mathrm{SH})$. The input beam width is $100 \mu \mathrm{m}$, and the pulse is propagating along the longitudinal $(z)$ axis focused over 5-mm focal length in argon at 1 atm pressure. In this classical, nonoptimized two-color setup the THz yield is about $1 \mu \mathrm{J}$. In Fig. 3(b) we shift the fundamental wavelength to $1600 \mathrm{~nm}$ and augment the SH ratio, which leads to a roughly twofold increase in the THz yield. Note that, by doubling the pump wavelength, only a factor 2 is achieved in the enhancement of the $\mathrm{THz}$ yield. This departs from the order of magnitude increase reported from setups with different focusing conditions in Refs. [16,23], but remains consistent with the nonmonotonic
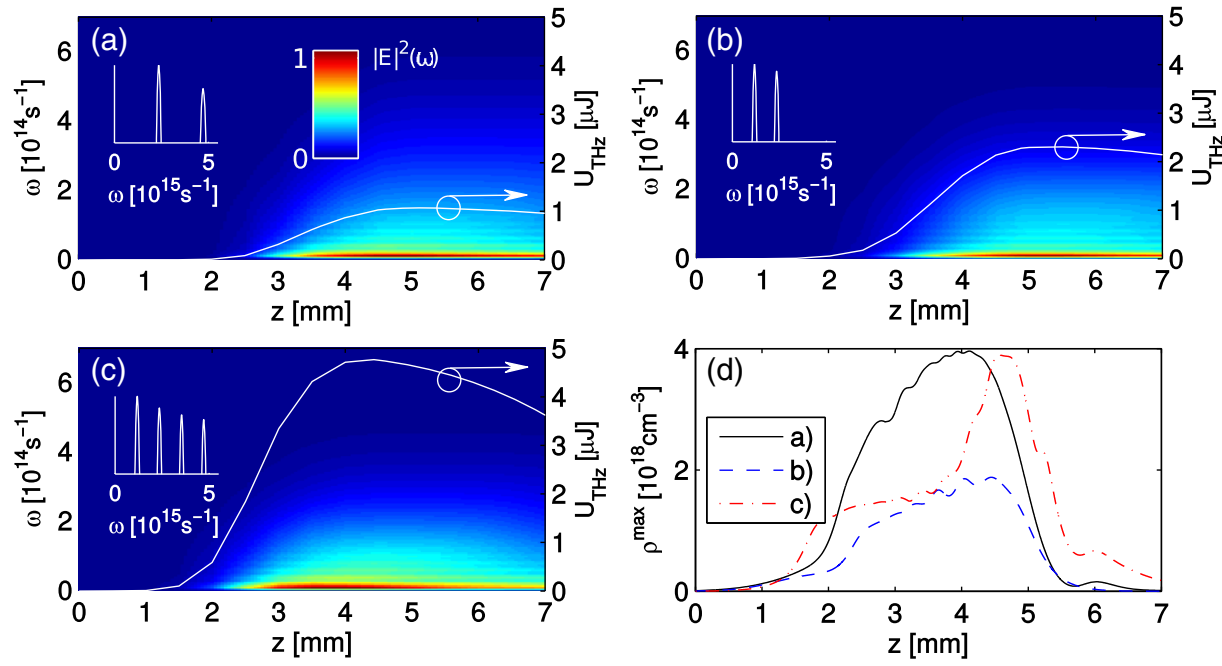

FIG. 3 (color online). Lowfrequency spectra (image plots) of (a) a two-color pulse for $\lambda_{0}=800 \mathrm{~nm}$ and its second harmonic in respective energy ratio of $\approx 0.06$, (b) a two-color pulse for $\lambda_{0}=1600 \mathrm{~nm}$ and its second harmonic in respective energy ratio of $\approx 0.4$, and (c) a four-color sawtooth pulse for $\lambda_{0}=1600 \mathrm{~nm}$ with the same energy ratio between the sum of harmonics and the fundamental. The overall $\mathrm{THz}$ energy $(\nu<100 \mathrm{THz})$ in the numerical box, $U_{\mathrm{THz}}(z)$, versus $z$ is shown by the right axes. The evolution of the peak free electron density for all three pulses is shown in (d), revealing a comparable fraction $(\approx 10 \%)$ of the neutral atoms ionized. 
increase of the $\mathrm{THz}$ field strength at high optical intensities $\left(>10^{14} \mathrm{~W} / \mathrm{cm}^{2}\right)$ predicted in [24]. In Fig. 3(c) the results for the four-color configuration are presented with pump pulses up to the fourth harmonic according to the sawtooth waveform. The THz yield is now $5 \mu \mathrm{J}$ [see Fig. 3(c)], i.e., 2.5 to 5 times larger than with the two-color cases in Figs. 3(a) and 3(b), which agrees with the LC predictions presented in Fig. 2. Finally, the electron density evolution for the three pulses of Figs. 3(a)-3(c) is shown in Fig. 3(d). Because pulse energy and focusing conditions are the same, the plasma densities exhibit similar dynamics. In particular, a comparable fraction $\approx 10 \%$ of the neutral atoms is ionized in all three cases, justifying the earlier assumption of a constant ionization yield in the local current model.

In conclusion, $\mathrm{THz}$ energy depends not only on the number of electrons ionized, but also on the pump field waveform. We have identified the free electron velocity as defined in Eq. (8) near the ionization instants as being crucial for optimizing the THz yield. By exploiting this insight, we have shown that the sawtooth waveform simultaneously maximizes this velocity and enhances the current asymmetry at the field extrema, which boosts $\mathrm{THz}$ conversion efficiency significantly compared to the standard two-color configuration. In setups where the effect of plasma defocusing can be neglected, the efficiency can increase by a factor of more than 50. However, taking into account intensity clamping, which should unavoidably occur when very high THz energy is targeted, the same factor is reduced by about 1 order of magnitude. We provided arguments showing that in the latter case the sawtooth shape is optimal. Comprehensive 3D simulations confirm this finding and reveal an efficiency of $2 \%$, which is unprecedented for $\mathrm{THz}$ generation in gases.

Numerical simulations were performed using high performance computing resources at Rechenzentrum Garching (RZG) and Mésocentre de Calcul Intensif Aquitain (MCIA). A. H. acknowledges the support of DFG (Project No. HU 1593/2-1). I. B. is thankful to P. Kinsler for useful discussions.

*Corresponding author. pedro.gonzalesdealaiza@cea.fr

[1] Sensing with Terahertz Radiation, edited by D. Mittleman (Springer, Berlin, 2002).

[2] M. Kreß, T. Löffler, M. D. Thomson, R. Dörner, H. Gimpel, K. Zrost, T. Ergler, R. Moshammer, U. Morgner, J. Ullrich et al., Nat. Phys. 2, 327 (2006).

[3] E. Pickwell and V. P. Wallace, J. Phys. D 39, R301 (2006).

[4] M. Tonouchi, Nat. Photonics 1, 97 (2007).

[5] B. Marx, Laser Focus World 43, 44 (2007).

[6] W. L. Chan, J. Deibel, and D. M. Mittleman, Rep. Prog. Phys. 70, 1325 (2007).

[7] M. C. Hoffmann and J. A. Fülöp, J. Phys. D 44, 083001 (2011).
[8] M. Woerner, W. Kuehn, P. Bowlan, K. Reimann, and T. Elsaesser, New J. Phys. 15, 025039 (2013).

[9] K.-L. Yeh, M. C. Hoffmann, J. Hebling, and K. A. Nelson, Appl. Phys. Lett. 90, 171121 (2007).

[10] C. Vicario, B. Monoszlai, and C. P. Hauri, Phys. Rev. Lett. 112, 213901 (2014).

[11] D. J. Cook and R. M. Hochstrasser, Opt. Lett. 25, 1210 (2000).

[12] K. Y. Kim, A. J. Taylor, J. H. Glownia, and G. Rodriguez, Nat. Photonics 2, 605 (2008).

[13] M. Thomson, M. Kreß, T. Löffler, and H. Roskos, Laser Photonics Rev. 1, 349 (2007).

[14] I. Babushkin, S. Skupin, and J. Herrmann, Opt. Express 18, 9658 (2010).

[15] I. Babushkin, S. Skupin, A. Husakou, C. Köhler, E. Cabrera-Granado, L. Bergé, and J. Herrmann, New J. Phys. 13, 123029 (2011).

[16] L. Bergé, S. Skupin, C. Köhler, I. Babushkin, and J. Herrmann, Phys. Rev. Lett. 110, 073901 (2013).

[17] M. Kress, T. Löffler, S. Eden, M. Thomson, and H. G. Roskos, Opt. Lett. 29, 1120 (2004).

[18] T. Bartel, P. Gaal, K. Reimann, M. Woerner, and T. Elsaesser, Opt. Lett. 30, 2805 (2005).

[19] K.-Y. Kim, J. H. Glownia, A. J. Taylor, and G. Rodriguez, Opt. Express 15, 4577 (2007).

[20] K. Reimann, Rep. Prog. Phys. 70, 1597 (2007).

[21] E. Cabrera-Granado, Y. Chen, I. Babushkin, L. Bergé, and S. Skupin, New J. Phys. 17, 023060 (2015).

[22] I. Babushkin, W. Kuehn, C. Köhler, S. Skupin, L. Bergé, K. Reimann, M. Woerner, J. Herrmann, and T. Elsaesser, Phys. Rev. Lett. 105, 053903 (2010).

[23] M.Clerici, M. Peccianti, B. E. Schmidt, L. Caspani, M. Shalaby, M. Giguère, A. Lotti, A. Couairon, F. Légaré, T. Ozaki, D. Faccio, and R. Morandotti, Phys. Rev. Lett. 110, 253901 (2013).

[24] A. Debayle, L. Gremillet, L. Bergé, and C. Köhler, Opt. Express 22, 13691 (2014).

[25] $v_{f}(t)$ is the velocity of a free electron injected at $t=-\infty$. The free electrons created by the photoionization process acquire different actual velocities, as they are injected at times $t_{n}$ with zero initial momentum.

[26] See Supplemental Material at http://link.aps.org/ supplemental/10.1103/PhysRevLett.114.183901 for detailed analytical calculations.

[27] Note that here we take into account the total current $J$, without neglecting $J_{A}$.

[28] L. Bergé, S. Skupin, R. Nuter, J. Kasparian, and J. P. Wolf, Rep. Prog. Phys. 70, 1633 (2007).

[29] L. E. Chipperfield, J. S. Robinson, J. W. G. Tisch, and J. P. Marangos, Phys. Rev. Lett. 102, 063003 (2009).

[30] S. Haessler, T. Balčiunas, G. Fan, G. Andriukaitis, A. Pugžlys, A. Baltuška, T. Witting, R. Squibb, A. Zaïr, J. W. G. Tisch, J. P. Marangos, and L. E. Chipperfield, Phys. Rev. X 4, 021028 (2014).

[31] M. Kolesik and J. V. Moloney, Phys. Rev. E 70, 036604 (2004).

[32] A. Dalgarno and A. E. Kingston, Proc. R. Soc. A 259, 424 (1960). 